\title{
A Escala de Humor de Brunel (Brums): Instrumento para Detecção Precoce da Síndrome do Excesso de Treinamento
}

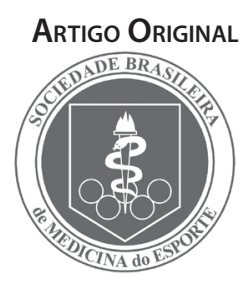

\section{Brunel Mood Scale (BRUMS): an Instrument for Early Detection of Overtraining Syndrome}

Izabel Cristina Provenza de Miranda Rohlfs $^{1}$

Tatiana Marcela Rotta' Caroline Di Bernardi Luft ${ }^{1}$

Alexandro Andrade ${ }^{1}$

Ruy Jornada Krebs'

Tales de Carvalho'

1. Universidade do Estado de Santa Catarina - Centro de Educação

Física, Fisioterapia e Desportos

- Florianópolis, SC.

Endereço para correspondência: Universidade do Estado de Santa Catarina - Centro de Educação Física, Fisioterapia e Desportos - Florianópolis - SC

Email: izabel.miranda@terra.com.br

Submetido em 05/07/2005 Versão final recebida em 03/11/2005 Aceito em 24/04/2006

\section{RESUMO}

A síndrome do excesso de treinamento tem uma grande relevância no âmbito esportivo, principalmente no que se refere a atletas de elite que buscam superar seus limites e àqueles que se submetem à prática de atividade física sem orientação especializada. O uso periódico do instrumento POMS, Perfil dos Estados de Humor, tem demonstrado eficácia na detecção de sinais iniciais da síndrome, prevenindo seu desenvolvimento completo. Em 2003, uma de suas versões abreviadas, passou a se denominar BRUMS, Escala de Humor de Brunel. O objetivo desse trabalho é apresentar a escala traduzida para a língua portuguesa juntamente com os dados obtidos em atletas brasileiros, referentes à primeira etapa do seu processo de validação no Brasil. O instrumento foi submetido ao método tradução-tradução reversa. A amostra foi composta por 34 atletas de futebol profissional, do gênero masculino, adolescentes e adultos. A aplicação do instrumento foi efetuada antes e depois de um treino com cargas moderadas e antes e depois de um jogo com cargas de alta intensidade. Para verificar a sensibilidade do BRUMS nas duas situações, foi realizado um teste t de student, entre pré e pós de cada variável, e aplicado uma correlação linear de Pearson, para verificar a relação entre os estados de humor. Os resultados indicaram que a raiva e a confusão mental reduziram significativamente $(p<0,05)$ no treino moderado (adolescentes) e que a depressão, vigor, fadiga e confusão mental aumentaram significativamente no jogo de alta intensidade (adultos). Para verificar a consistência do resultado obtido pelo BRUMS foi realizada uma entrevista semi estruturada junto aos atletas. Os relatos dos atletas em relação às situações de treino e jogo ajudaram a explicar os resultados do BRUMS. Nessa primeira etapa da validação, o instrumento BRUMS mostrou-se sensível e fidedigno na avaliação de estados de humor.

Palavras-chave: desempenho desportivo, estados de humor, mensuração, escalas.

\begin{abstract}
The overtraining syndrome has a great relevance in the sportive scope, mainly as for elite athletes who search to transcend their limits and for those who submit to physical activity practice without specialized orientation. The periodic use of the POMS instrument, Profile of Mood States, has demonstrated effectiveness in the detection of the syndrome's initial signals, preventing its complete development. In 2003, one of its shortened versions, started to be called BRUMS, Brunel Mood Scale. The objective of this work is to present the scale translated into Portuguese language together with the data from Brazilian athletes referring to the first stage of its validation process in Brazil. The instrument was submitted to the translationback translation method. The sample was composed for 34 male professional soccer players, adolescents and adults. The application of the instrument was conducted before and after training with moderate loads and before and after a game with high intensity loads. In order to verify the sensibility of the BRUMS in both situations, a t -student test was performed between before and after each variable and Pearson linear correlation was applied to verify the relationship between the moods. The results have indicated that anger and mental confusion significantly reduced $(p<0.05)$ in moderate training (adolescents) and depression, vigor, fatigue and mental confusion have significantly increased in the high intensity game (adults). In order to verify the consistency of the result gotten by BRUMS a semi-structuralized interview was conducted with the athletes. The athletes' reports concerning training and game situations helped to explain the results of the BRUMS. In this first stage of the validation, the BRUMS instrument was sensitive and trustworthy in the moods evaluation.
\end{abstract}

Keywords: physical performance, mood states, measurament, scales. 


\section{INTRODUÇÃO}

Os métodos de investigação e medidas psicológicas no esporte e na atividade física eram condicionados aos métodos aplicados na psicologia geral. Os instrumentos utilizados buscavam uma avaliação clínica de fenômenos psicológicos (traços, atitudes, habilidades), mas eram desvinculados do contexto do esporte ou atividade física que se queria avaliar ${ }^{(1,2)}$. Com o crescente desenvolvimento da Psicologia Cognitiva e da Psicologia Social, os psicólogos do esporte enfatizaram a avaliação psicológica em variáveis de caráter sócio-psicológicas, estudando autopercepções de estresse, motivação, facilitação social, grupo esportivo e cognições ${ }^{(3-5)}$.

A partir deste desenvolvimento, os métodos de avaliação psicológica aliados à prática do treinamento esportivo obtiveram resultados expressivos, mensurando características, atributos e estados emocionais transitórios sob condições controladas, desenvolvendo perfis e prognósticos. O objetivo da psicometria passou a ser a promoção e prevenção da saúde de indivíduos envolvidos em programas de treinamento e atividade física, atingindo, assim, a melhora do desempenho esportivo dos mesmos.

Nos últimos 50 anos, observou-se uma série de mudanças sociais e ambientais que fizeram com que atividade física passasse a ser considerada fator de prevenção e tratamento de várias doenças. A criação de programas de atividade física e saúde, que no Brasil foi a partir dos anos 90, e a atenção dada pelos serviços de saúde ao controle do sedentarismo incentivaram a busca da qualidade de vida e a prática regular de exercícios ${ }^{(6,7)}$. Esses fatores aliados à mudança de padrões estéticos têm levado indivíduos a buscarem, através do exercício físico, a redução da massa corporal, o aumento da massa muscular e do condicionamento aeróbio. Por outro lado, a grande exigência do esporte competitivo tem provocado sérias conseqüências em atletas envolvidos em treinamento de alto nível. Constata-se que atletas e não atletas podem desenvolver a síndrome do excesso de treinamento (overtraining), que resulta do desequilíbrio entre a demanda do exercício e a possibilidade de assimilação de treinamento, acarretando alterações metabólicas, com conseqüências que abrangem não apenas o desempenho, mas também outros aspectos fisiológicos e emocionais ${ }^{(8)}$.

A síndrome do excesso de treinamento afeta uma considerável porcentagem de indivíduos envolvidos em programas de treinamento intensivo. Estima-se em 7\% a 20\% por temporada a sua incidência em atletas $^{(9-11)}$. Atletas em todos os níveis de performance podem desenvolver essa síndrome e um relevante número de sinais e sintomas tem sido associado à mesma. Fry et al. ${ }^{(12)}$, listou mais de 200 em sua revisão sobre overtraining em 1991 ${ }^{(13)}$. Porém, apesar dessa extensa lista, ainda não existem critérios diagnósticos bem estabelecidos ${ }^{(14)}$, talvez até pela falta de uma cultura que implique sistemática e rotineira avaliação dos desportistas.

Seu mecanismo bioquímico-metabólico ainda permanece desconhecido ${ }^{(15)}$. O único tratamento efetivo é o repouso prolongado e a melhor maneira de se evitar a manifestação da síndrome é a prevenção. Como ainda não existem marcadores fisiológicos ou biológicos objetivos que permitam um diagnóstico precoce do quadro(16), o uso de instrumentos que possibilitam medidas de estados de humor tem demonstrado eficácia na detecção de sinais iniciais da síndrome do excesso de treinamento, prevenindo seu desenvolvimento completo ${ }^{(11,17)}$.

Um dos instrumentos psicológicos que tem sido efetivo e sensível em detectar sinais que antecedem a manifestação da síndrome do excesso de treinamento(18) é o POMS, "Perfil dos estados de Humor"(1). Observou-se, por meio de alguns estudos ${ }^{(19)}$, que o fator fadiga elevado equiparado ao fator depressão elevado, apresentado no gráfico individual do POMS, parece ser o produto do excesso de treinamento, e os fatores depressão, confusão mental e raiva acima do escore padrão, são considerados como estados de alteração emocional. Uma das versões abreviadas do POMS foi validada para o uso em adolescentes (POMS-A) ${ }^{(20)}$, e posteriormente passou por um rigoroso processo de validação para avaliação em adultos (21), passando a se denominar BRUMS, Escala de Humor de Brunel. A revisão do artigo original foi recentemente publicada por Rohlfs et al. ${ }^{(8)}$.

Estes instrumentos são citados por Peter C. Terry como forma de intervenção em psicologia do esporte e da saúde a partir do controle geral da disposição mental do indivíduo (objetivando a melhora constante do humor do avaliado), como catalisadores para discussão durante as sessões individuais, para a identificação precoce de problemas, controle do humor de capitães de time e da equipe de apoio, controle da carga de treinamento e de respostas emocionais à lesão, para a previsão de desempenho (sem o objetivo de selecionar atletas), para monitorar a aclimatação, fadiga de viagens e reabilitação de overtraining ou de lesões em atletas e como um fator contribuinte para a individualização do treinamento(22,23).

Portanto, o uso destes instrumentos pode ser ampliado para populações de não atletas, tanto na disposição da prática de exercícios físicos, contribuindo na organização e planificação de cargas de treinamento, quanto no controle do estresse em indivíduos participantes de programas de atividade física e reabilitação, abrangendo uma faixa etária de adolescentes a adultos ${ }^{(24)}$.

Para que um instrumento de avaliação psicológica seja utilizado, é necessário passar por uma série de análises estatísticas, a fim de agrupar ou estabelecer os itens que se referem às dimensões das categorias mensuradas. Após a avaliação das propriedades psicométricas da escala, questionário ou teste, a escolha da unidade de análise irá depender do objetivo da avaliação(8).

No esporte e na atividade física e saúde são poucos os instrumentos em Psicologia no Brasil com especificidade e aplicabilidade no contexto de atuação profissional que fazem uma leitura do fenômeno que se objetiva avaliar. O BRUMS, em seu estudo de validação(21), foi considerado um instrumento apropriado para avaliação de perfis do humor, além do que a brevidade da escala facilita a coleta de dados em ambientes de pesquisa ${ }^{(8)}$.

Diante da perspectiva da utilização de um instrumento que demonstre sensibilidade e especificidade na detecção da síndrome do excesso de treinamento, este artigo visa apresentar, de forma sucinta, a Escala de Humor de Brunel, o BRUMS, em sua versão na língua portuguesa, juntamente com os dados de atletas brasileiros referentes à primeira etapa do seu processo de validação. O estudo completo está sendo realizado no Brasil também em população de não atletas.

\section{Aspectos teóricos relacionados à validação de instrumentos}

A validade é considerada a característica mais importante na avaliação de uma escala, e é realizada por meio do acúmulo de evidências que suportam as inferências feitas de acordo com resultados obtidos pelas mesmas. Ela pode ser verificada sob diferentes perspectivas, enfocando-se o construto, o conteúdo e/ou o critério.

A validade do construto refere-se à demonstração de que o instrumento realmente mede aquilo que tenciona medir(21). Pasquali(25) considera a validade de construto como primordial, já que confirma ou rejeita os pressupostos teóricos eleitos para a construção do instrumento e aponta que a teoria deve fundamentar qualquer empreendimento científico e, com isso, deve ser clara a explicitação da teoria sobre a qual o teste foi construído.

A análise da validade de conteúdo visa verificar o que o teste pretende medir. Para a validação do conteúdo não existem métodos 
estatísticos refinados, normalmente recorre-se à análise de juízes experientes na área, para avaliarem adequadamente as propriedades do instrumento ${ }^{(26)}$.

No que se refere à validade de critério, os resultados obtidos serão expressos em graus de concordância - identidade entre o teste e a outra forma - denominada de relação entre eles. Ela avalia o grau com que o instrumento discrimina entre pessoas que se diferem em determinada(s) característica(s) de acordo com um critério padrão e é concebida como o grau de eficácia para predizer um determinado desempenho do sujeito, que deve ser medido por meio de técnicas independentes do próprio teste(26)

Deve-se também avaliar a confiabilidade do instrumento que geralmente é utilizado para se referir à reprodução de uma medida, ou seja, o grau de concordância entre múltiplas medidas de um mesmo objeto (27). A avaliação da confiabilidade de um instrumento é feita através da comparação de diversas aplicações do instrumento ao mesmo indivíduo.

Contudo, é importante ressaltar que a percepção subjetiva do pesquisado é fundamental para a compreensão das interpretações dos resultados, pois a realidade investigada é a realidade percebida subjetivamente pelo mesmo, como síntese de múltiplas determinações, que influenciam diretamente seu estilo de vida, saúde e seu desempenho desportivo e na vida em geral ${ }^{(28)}$. Não é possível avaliar o julgamento subjetivo com métodos estatísticos, mas somente com a avaliação do investigador em julgar se o instrumento afere o que deveria aferir(25). Nesse sentido, para a avaliação psicológica, faz-se necessária a utilização de métodos e medidas quantitativas e qualitativas como ferramentas no reconhecimento de processos psicológicos (emoções, cognições, alterações psicofisiológicas) que intervêm no desempenho do indivíduo.

\section{POMS - "profile of mood states"}

O POMS é um instrumento de avaliação dos estados de humor, que contém 65 itens e mede seis fatores de humor: tensão, depressão, raiva, vigor, fadiga e confusão mental. Esse teste foi desenvolvido, a partir do final da década de 50, para a observação de estados em diferentes momentos de flutuação de humor em pacientes psiquiátricos por Mcnair, Lorr, e Droppleman ${ }^{(1)}$.

A divisão das seis subescalas é a seguinte: "Tensão", contém 9 itens; "Depressão", 15 itens, "Raiva", 12 itens; "Vigor", 8 itens, "Fadiga", 7 itens e "Confusão", com 7 itens. Originalmente os sete itens restantes faziam parte de um sétimo fator, "Amabilidade", que foi descartado por não apresentar validade e confiabilidade adequadas, mas os itens permaneceram a fim de não haver alteração no inventário. Com a soma dos seis fatores, considerando "Vigor" um valor negativo, obtém-se uma medida total de alteração de humor ${ }^{(17)}$.

A tradução e a validação do POMS na versão em português foram feitas recentemente em um estudo sobre alterações de humor associadas à atividade física ${ }^{(17)}$.

\section{A Escala de Humor de Brunel (BRUMS)}

A Escala de Humor de Brunel, BRUMS, foi desenvolvida para permitir uma rápida mensuração do estado de humor de populações compostas por adultos e adolescentes. Adaptado do "Profile of Mood States"(1), o BRUMS contém 24 indicadores simples de humor, tais como as sensações de raiva, disposição, nervosismo e insatisfação que são perceptíveis pelo indivíduo que está sendo avaliado. Os avaliados respondem como se situam em relação às tais sensações, de acordo com a escala de 5 pontos (de 0 = nada a 4 = extremamente). A forma colocada na pergunta é "Como você se sente agora", embora outras formas: "Como você tem se sentido nesta última semana, inclusive hoje", ou "Como você normalmente se sente" possam ser usadas. O BRUMS leva cerca de um a dois minutos para ser respondido.

Os 24 itens da escala compõem as seis subescalas: raiva, confusão, depressão, fadiga, tensão e vigor. Cada subescala contém quatro itens. Com a soma das respostas de cada subescala, obtém-se um escore que pode variar de 0 a 16.

O fator tensão refere-se à alta tensão músculo-esquelética, que pode não ser observada diretamente ou por meio de manifestações psicomotoras: agitação, inquietação, etc ${ }^{(18)}$.

A depressão representa um estado depressivo, onde a inadequação pessoal se faz presente, indicando humor deprimido e não depressão clínica. Representa sentimentos como autovalorização negativa, isolamento emocional, tristeza, dificuldade em adaptação, depreciação ou auto-imagem negativa ${ }^{(29)}$.

A raiva descreve sentimentos de hostilidade a partir de estados de humor relacionados à antipatia em relação aos outros e a si mesmo. Estado emocional que varia de sentimentos de leve irritação até a cólera associada a estímulos do sistema nervoso autônomo ${ }^{(30)}$.

O fator vigor caracteriza estados de energia, animação e atividade, elementos essenciais para o bom rendimento de um atleta, já que indica um aspecto humoral positivo. Caracterizado por sentimentos de excitação, disposição e energia física, é relacionado a outros fatores de forma inversa ${ }^{(18)}$.

No que se refere à fadiga, esta representa estados de esgotamento, apatia e baixo nível de energia. Os sintomas da fadiga crônica são descritos com alterações gradativas na atenção, concentração e memória; também nos distúrbios de humor, irritabilidade e posteriormente às alterações de sono, cansaço físico, repercutindo-se no processo de iniciação de problemas de ordem psicossomática, fisiológica e psíquica $^{(31)}$.

O fator confusão mental pode ser caracterizado por atordoamento, situando-se, possivelmente, como uma resposta/resultado à ansiedade e à depressão. Sentimentos de incerteza, instabilidade para controle de emoções e atenção(29).

Tendo sido respondidas a todas as palavras que pertencem às variáveis de uma amostra, esse número de variáveis torna-se um conjunto de itens de uma escala de avaliação (raiva, fadiga, vigor, depressão, tensão, confusão mental). Utiliza-se o valor de cada item com base no escore do teste e, finalmente, o resultado é representado em um gráfico, caracterizando o estado emocional atual do aplicando. Há separação dos dados de cada diferente população: estudantes adultos (> 18 anos de idade), atletas adultos (> 18 anos de idade), adolescentes escolares (12 a 17 anos), jovens atletas (12 a 17 anos) (24).

Todas as normas foram geradas de uma coleta de dados originada da pergunta que teve como forma "Como você se sente agora?" e podem não ser aplicáveis a dados coletados usando outras formas. Para os estudantes e adolescentes escolares, as normas foram geradas a partir de dados coletados na sala de aula. Normas para atletas foram geradas a partir de dados coletados aproximadamente uma hora antes de competição. Escores brutos podem ser avaliados em planilhas próprias. Há diferentes planilhas para estudantes adultos, atletas adultos, estudantes adolescentes e atletas adolescentes ${ }^{(24)}$.

\section{MÉTODO}

O método de tradução-tradução reversa é um processo estruturado, cuja finalidade é a obtenção de um instrumento equivalente ao original, mas adaptado à nossa cultura, no qual se busca uma equivalência conceitual nos termos utilizados e, principalmente, se valoriza a pertinência dos itens em nosso contexto cultural. Uma das principais vantagens advindas da aplicação deste método é permitir 
a comparação dos resultados obtidos ao utilizar a versão adaptada e nosso meio com os obtidos por outros investigadores com a versão original ou com a escala traduzida para a outra língua sempre que se utiliza este processo de tradução-tradução reversa. Com ele podemos medir um mesmo fenômeno em diferentes culturas com o objetivo de identificar diferenças atribuíveis ao contexto cultural e não a diferenças no instrumento utilizado ${ }^{(32)}$.

O instrumento original foi submetido à tradução para o português e à tradução reversa da versão conseguida para o inglês (back translation) por dois tradutores especializados e independentes. A versão ajustada após a tradução reversa foi avaliada por dois especialistas bilíngües da área da psicologia que, após consenso, definiram a versão em português.

Com o intuito de avaliar a viabilidade e efetividade da tradução e a adaptação do instrumento para os parâmetros culturais brasileiros, efetuou-se a primeira testagem do instrumento em 30 atletas de triatlon, adolescentes e adultos, após uma competição. Essa testagem do instrumento em população brasileira possibilitou a revisão cuidadosa dos 24 itens realizada por um dos autores do instrumento, Peter C. Terry, a partir dos escores apresentados no gráfico individual dos atletas testados e das dificuldades apontadas por eles com relação aos termos presentes nos itens. Após essa revisão, a escala passou por uma comissão de especialistas que levantou a possibilidade de troca da tradução de 12 dos itens que tiveram uma contribuição pequena para a consistência interna da escala. Estes só foram trocados depois que se encontrou outra palavra que pudesse servir à tradução.

Com essa versão ajustada realizou-se um estudo piloto com 34 atletas do gênero masculino de futebol de um clube de primeira divisão do campeonato brasileiro, dividido em duas categorias: adolescentes (16 e 17 anos) e adultos (19 e 20 anos). Após a explanação dos objetivos do estudo, todos assinaram o termo de consentimento livre e esclarecido. A aplicação do BRUMS foi efetuada antes e depois de um treino com cargas moderadas no grupo dos adolescentes e antes e depois de uma situação de jogo com cargas altas no grupo dos adultos.

Para verificar a sensibilidade do instrumento para detectar as mudanças ocorridas em ambas as situações (treino e jogo), aplicou-se após o jogo do grupo de adultos e o treino do grupo de adolescentes uma entrevista semi-estruturada com perguntas referentes à percepção dos atletas com relação ao jogo/treino. As entrevistas foram analisadas por meio da técnica de análise de conteúdo de Bardin ${ }^{(33)}$.

Foi realizado um teste t de student, entre cada variável, considerando-se o pré e o pós-teste e foi aplicado um teste de correlação parcial, o cálculo de correlação linear de Pearson, controlado pela fase (adolescente/adulto), porque além da amostra abranger idades (categorias) diferentes, a coleta foi feita no grupo dos adolescentes em uma situação de treino com cargas moderadas e, no grupo dos adultos, em uma situação de jogo com cargas intensas.

\section{RESULTADOS}

A tabela 1 apresenta as variáveis: tensão, depressão, raiva, vigor, fadiga e confusão mental na categoria de atletas adolescentes durante um treino com cargas moderadas, indicando diferenças significativas $(p<0,05)$ nos fatores raiva e confusão mental. A raiva, no treino leve, diminuiu significativamente, enquanto que no jogo aumentou significativamente.

Os fatores confusão mental e raiva no grupo dos adolescentes diminuíram significativamente após o treino com cargas moderadas indicando que o mesmo não provocou alterações significativas nesses estados de humor, ou seja, como não houve nenhuma situação ansiogênica que pudesse causar atordoamento aos atletas adolescentes, o treino
Tabela 1. Estados de Humor dos adolescentes antes e após um treino com cargas moderadas

\begin{tabular}{c|c|c|c|c|c}
\hline \multirow{2}{*}{ Estados de Humor } & \multicolumn{2}{|c|}{ Pré-Teste } & \multicolumn{2}{c|}{ Pós-Teste } & \multirow{2}{*}{ p } \\
\cline { 2 - 5 } & Média & DP & Média & DP & \\
\hline Tensão & 2,90 & 3,29 & 2,55 & 2,52 & 0,051 \\
\hline Depressão & 3,70 & 4,49 & 2,60 & 4,02 & 0,057 \\
\hline Raiva & 2,70 & 3,70 & 1,55 & 3,52 & $0,01^{*}$ \\
\hline Vigor & 8,95 & 2,56 & 8,80 & 2,26 & 0,78 \\
\hline Fadiga & 3,05 & 2,54 & 2,95 & 2,78 & 0,85 \\
\hline Confusão & 3,15 & 2,81 & 2,05 & 2,54 & $0,003^{*}$ \\
\hline
\end{tabular}

*diferença estatisticamente significativa ao nível de $p<0,05$

DP: desvio padrão

moderado pôde até mesmo descontraí-los, indicando também a sensibilidade do BRUMS à intensidade do estímulo aplicado. Os atletas indicaram, nas entrevistas, que o treinador esclareceu ao grupo de atletas quem iria jogar o próximo jogo após o término do treino e antes da aplicação do instrumento BRUMS. Provavelmente, essa informação minimizou os sentimentos de nervosismo e preocupações em relação à atuação no próximo jogo, o que pode ser verificado no teste quando se observou redução na raiva e na confusão mental. Ou seja, ter a informação acerca da lista de atletas escalados pode minimizar a confusão mental apresentada pelo atleta, pois, se escalado, a ameaça de ser dispensado ou de não estar rendendo fica reduzida.

A tabela 2 apresenta as variáveis: tensão, depressão, raiva, vigor, fadiga e confusão mental na categoria de atletas adultos durante um jogo com alta carga, indicando diferenças significativas $(p<0,05)$ nos fatores depressão, raiva, vigor e fadiga.

Tabela 2. Estados de Humor dos adultos antes e após um jogo com cargas intensas

\begin{tabular}{c|c|c|c|c|c}
\hline \multirow{2}{*}{ Estados de Humor } & \multicolumn{2}{|c|}{ Pré-Teste } & \multicolumn{2}{c}{ Pós-Teste } & \multirow{2}{*}{ p } \\
\cline { 2 - 5 } & Média & DP & Média & DP & \\
\hline Tensão & 2,81 & 2,04 & 3,44 & 2,28 & 0,145 \\
\hline Depressão & 0,94 & 0,99 & 4,75 & 3,70 & $0,001^{*}$ \\
\hline Raiva & 1,00 & 1,37 & 4,88 & 4,75 & $0,003^{*}$ \\
\hline Vigor & 13,40 & 2,22 & 7,88 & 3,95 & $0,000^{*}$ \\
\hline Fadiga & 1,88 & 1,67 & 5,88 & 3,95 & $0,000^{*}$ \\
\hline Confusão & 1,69 & 2,09 & 3,44 & 3,03 & 0,053 \\
\hline
\end{tabular}

* diferença estatisticamente significativa ao nível de $p<0,05$ DP: desvio padrão

Os fatores raiva e depressão no grupo dos adultos: a partir da análise dos dados qualitativos, a raiva é atribuída pelos atletas à situação do resultado final do jogo, que, segundo os mesmos, era representativo naquele momento do treinamento, pois os atletas perderam a partida. O aumento significante no fator raiva pode indicar que o instrumento BRUMS é sensível para detecção de estados alterados de humor em situações tensas. $O$ estado de depressão é também atribuído pe- 
los atletas por um sentimento de tristeza à derrota vivenciada pelo mesmo grupo. Partindo de avaliações periódicas efetuadas durante o treinamento esportivo de tal grupo, pode-se elaborar programas de treinamento que enfatizem variáveis no trabalho de auto-confiança e imagem positiva dos atletas no desempenho esportivo.

O fator vigor e fadiga nos atletas adultos: os atletas foram questionados em relação à alteração de vigor, devido ao índice no gráfico ter diminuído depois da situação de jogo e à fadiga que teve seu índice aumentado. Os mesmos afirmaram: "foi o cansaço do jogo", "foi o desânimo por ter perdido", "tava cansado de tanto correr". Infere-se que ocorrem duas variáveis distintas de reação ao fator fadiga: o cansaço físico e o desânimo. Sendo a primeira uma atribuição fisiológica e a segunda emocional. O fator vigor se comportou de maneira inversa à fadiga, diminuindo significativamente e indicando perda de disposição e energia física devido à perda do jogo e ao cansaço físico.

Deve-se observar também que as maiores diferenças ocorreram nos adultos na situação de jogo com cargas elevadas indicando sensibilidade do instrumento à intensidade do estímulo e às mudanças, pois as maiores diferenças foram verificadas na situação mais tensa.

Essas diferenças indicam a alta sensibilidade do BRUMS para detectar as alterações nos estados de humor em diferentes situações, pois poucos estados de humor foram alterados, e aqueles que modificaram, foram explicados pela entrevista. No entanto, quando o estímulo foi intenso o suficiente para produzir mudanças significativas nos estados de humor, o BRUMS foi sensível a ponto de detectar mudanças mais significativas nos estados de humor específicos e confirmados pelas entrevistas dos atletas, que perderam o jogo.

Ao aplicar as correlações entre os estados de humor pré e pós-teste, moderados pela fase, verificou-se que a tensão foi correlacionada positivamente com as variáveis de pré-teste de depressão $(r=0,54 / p=0,001)$, raiva $(r=0,69 / p<0,000)$ e confusão $(r=0,512 / p=0,002)$ e fadiga $(r=0,37)$ $\mathrm{p}=0,027)$. $\mathrm{O}$ fator fadiga só correlacionou com a tensão no pré-teste, no pós-teste não houve correlação. No pós-teste, a tensão se correlacionou com depressão $(r=0,60 / p<0,000)$, raiva $(r=0,48 / p=0,003)$ e

No grupo de atletas adultos, a depressão além de se correlacionar positivamente com a tensão, foi correlacionada no pré-teste com raiva $(r=0,711 / p<0,000)$, vigor $(r=-0,35 / p=0,041)$, fadiga $(r=0,61 / p<0,000)$ confusão $(r=0,70 / p<0,000)$. No pós-teste, a depressão se correlacionou com raiva $(r=0,76 / p<0,000)$, e com confusão $(r=0,64 / p<0,000)$. No pós-teste não foram verificadas correlações da depressão com vigor

A raiva foi correlacionada positivamente com fadiga $(r=0,40 /$ $p=0,017)$, com a confusão $(r=0,55 / p=0,001)$ e com a tensão e a depressão (os valores estão descritos acima). No pós-teste a raiva foi correlacionada positivamente com os mesmos estados do pré-teste, fadiga $(r=0,49 / p=0,002)$, confusão $(r=0,48 / p=0,003)$ e tensão e depressão.

O fator vigor apresentou correlação negativa apenas com o fator depressão $(r=-0,35 / p=0,041)$ e fadiga $(r=-0,43 / p=0,010)$. Este dado está de acordo com Terry et al. ${ }^{(18)}$ que indica que o fator vigor pode se correlacionar apenas com os fatores depressão e fadiga.

A fadiga apresentou, no pré-teste, correlação com os itens destacamental $(r=0,41 / p=0,013)$. No pós-teste, a fadiga não apresentou correlação com confusão mental, mas manteve as correlações do pré-teste. Todas essas correlações indicam o grau em que cada variável está relacionada. No entanto deve-se destacar que embora estas relações possam ser observadas para garantir a sensibilidade do instrumento, elas não devem ser perfeitas, ou seja, apresentar um coeficiente de corem um estado de calma e estabilidade (treino com cargas moderadas) confusão $(r=0,65 / p<0,000)$. e com fadiga. dos anteriormente, tensão, depressão, raiva e também com confusão

relação (r) muito próximo de 1 ou -1. Isso não deve acontecer porque uma correlação perfeita pode indicar que o instrumento não capta as nuanças que diferenciam os estados de humor, e sim, mensura uma tendência de estresse geral. Por exemplo, ao testar a ansiedade, por meio do IDATE e a depressão, por meio do Inventário de Beck, Andrade e Goreinstein ${ }^{(34)}$ encontraram correlações perfeitas entre os resultados dos dois testes, indicando que os instrumentos não são tão sensíveis e específicos na mensuração da ansiedade e da depressão (que são conceitualmente diferentes).

A escala está sendo submetida a um estudo de confiabilidade tipo teste-reteste com estudantes e atletas adolescentes e adultos avaliados em momentos diferentes, cujo resultado será utilizado nas próximas etapas necessárias para a conclusão do estudo de validação do instrumento. A versão traduzida para a língua portuguesa é apresentada a seguir.

\section{VERSÃO TRADUZIDA DO BRUMS PARA A LÍNGUA PORTUGUESA}

\section{A Escala de Humor de Brunel (BRUMS)}

Abaixo está uma lista de palavras que descrevem sentimentos. Por favor, leia tudo atenciosamente. Em seguida assinale, em cada linha, o quadrado que melhor descreve COMO VOCÊ SE SENTE AGORA. Tenha certeza de sua resposta para cada questão, antes de assinalar.

\section{Escala:}

$\begin{array}{ll}0=\text { nada } & 1=\text { um pouco } \quad 2 \text { = moderadamente } \\ 3=\text { bastante } & 4=\text { extremamente }\end{array}$

\begin{tabular}{|c|c|c|c|c|c|}
\hline & 0 & 1 & 2 & 3 & 4 \\
\hline 1. Apavorado & $\square$ & $L$ & $\square$ & L & 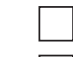 \\
\hline 2. Animado & $\square$ & & 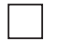 & & L \\
\hline 3. Confuso & $\square$ & & - & L & L \\
\hline 4. Esgotado & & & $L$ & & 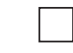 \\
\hline 5. Deprimido & & & 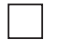 & L & 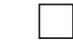 \\
\hline 6. Desanimado & & & [ & 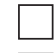 & 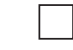 \\
\hline 7. Irritado & & & - & L & 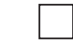 \\
\hline 8. Exausto & & & - & $L$ & L \\
\hline 9. Inseguro & 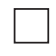 & & - & 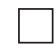 & $\square$ \\
\hline 10. Sonolento & & & $\square$ & $\square$ & $\square$ \\
\hline 11. Zangado & $\square$ & & $\square$ & $\square$ & $\square$ \\
\hline 12. Triste & & & 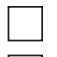 & 5 & $\square$ \\
\hline 13. Ansioso & & & 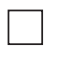 & L & $\square$ \\
\hline 14. Preocupado & & & L & $L$ & L \\
\hline 15. Com disposição & ¿ & & 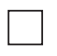 & L & ¿ \\
\hline 16. Infeliz & L & & L & L & L \\
\hline 17. Desorientado & 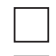 & & [ & 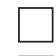 & $\square$ \\
\hline 18. Tenso & $\square$ & & 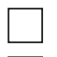 & $\square$ & $\square$ \\
\hline 19. Com raiva & $\square$ & $\square$ & L & $\square$ & $\square$ \\
\hline 20. Com energia & $\square$ & $\square$ & $\square$ & $\square$ & $\square$ \\
\hline 21. Cansado & $\square$ & & ए & $\square$ & $\square$ \\
\hline 22. Mal-humorado & $\square$ & $\Gamma$ & L & $\square$ & $\square$ \\
\hline 23. Alerta & $\square$ & & $\square$ & $\square$ & $\square$ \\
\hline 24. Indeciso & $\square$ & $\square$ & $\square$ & $\square$ & $\square$ \\
\hline
\end{tabular}




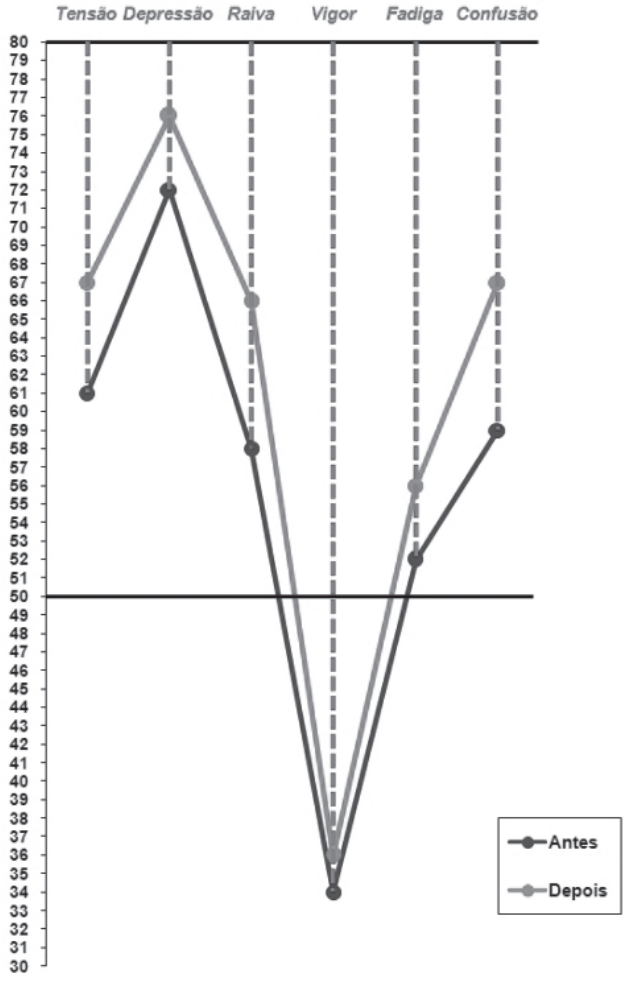

Figura 1. Gráfico individual do BRUMS de atleta apresentando alteração no estado emocional

\section{CONCLUSÕES}

A leitura, a partir da avaliação do indivíduo sobre o seu perfil de estado de humor, apresentando no gráfico diferentes variáveis interligadas para confirmação de alteração emocional, é um meio eficaz para avaliar o distresse associado ao excesso de treinamento. A aplicabilidade da medida resulta em sinais de alteração de humor, como também a leitura do gráfico conjuntamente com o acompanhamento periódico dos indivíduos na complementação dos dados de cargas de treinamento na prática da atividade física, ou no esporte competitivo, objetivam a prevenção da saúde física e psicológica dos indivíduos envolvidos.

Nessa primeira etapa da validação, o instrumento BRUMS mostrouse sensível e fidedigno na avaliação dos estados emocionais alterados em atletas, propiciando uma intervenção psicológica efetiva no processo de treinamento e na rotina diária dos avaliados, possibilitando a elaboração de programas que considerem o princípio da individualidade na planificação do treinamento e o encaminhamento clínico de estados emocionais que necessitem de acompanhamento.

O processo de tradução do instrumento BRUMS para o português, apresentado nesse estudo, adota a metodologia utilizada em qualquer instrumento no qual objetiva-se a tradução em diferentes línguas. Confirma, assim, a necessidade prática de uma escala que forneça parâmetros psicométricos que referenciem o contexto para o qual o instrumento será utilizado, aproximando-se da realidade objetiva que se quer ler, a partir da percepção daquele que a lê. Portanto torna-se relevante, além do tratamento estatístico dos dados coletados, a leitura qualitativa junto à população pesquisada, visando a validade e precisão do instrumento utilizado na investigação.

As diferentes mudanças encontradas em situações esportivas distintas indicam que o BRUMS é sensível para verificar os estados de humor em diversas situações. As entrevistas reforçaram ainda mais essa sensibilidade. As correlações entre os estados de humor verificadas neste estudo permitem concluir que o BRUMS detecta os estados de humor de forma diferenciada, e não apenas uma medida de distresse ou bem estar geral.

Estudos em andamento visam realizar as etapas seguintes para a validação do instrumento BRUMS na detecção de estados alterados de humor em atletas e não atletas brasileiros, adolescentes e adultos, de acordo com as condições e características nacionais.

Todos os autores declararam não haver qualquer potencial conflito de interesses referente a este artigo.

\section{REFERÊNCIAS BIBLIOGRÁFICAS}

1. Mcnair DM, Lorr M, Droppleman LF. Manual for the profile of mood states. San Diego, CA: Educational and Industrial Testing Services; 1971.

2. Brandão MR. Fatores de stress em jogadores de futebol profissional [tese].Campinas (SP): Universidade estadual de Campinas; 2000.

3. Salmuski D. Psicologia do esporte: teoria e aplicação prática. Belo Horizonte: Universidade Federal de Minas Gerais; 1995.

4. Brandão MR. Equipe nacional de voleibol masculino: um perfil sócio-psicológico à luz da ecologia do desenvolvimento humano [dissertação]. Santa Maria (RS): Universidade Federal de Santa Maria; 1996.

5. Becker Jr B. Manual de psicologia do esporte e exercício. Porto Alegre: Nova Prova; 2000.

6. Carvalho T. Sedentarismo o inimigo público nำ1. Rev Bras Med Esporte 1999; 5: xi-xii._

7. American College of Sports Medicine. ACSM's Guidelines for exercise testing and prescription. 6th ed. Baltimore: Williams \& Wilkins; 2000.

8. Rohlfs ICPM, Carvalho T, Rotta TM, Krebs RJ. Aplicação de instrumentos de avaliação de estados de humor na detecção da síndrome do excesso de treinamento. Rev Bras Med Esporte 2004; 10: 111-6.

9. Morgan WP, Brown DR, Raglin JS, O'Connor PJ, Ellickson KA. Psychological monitoring of overtraining and staleness. Br J Sports Med 1987; 25: 107-14

10. Hooper SL, Mackinnon LT, Gordon RD, Bachmann AW. Hormonal responses of elite swimmers to overtraining. Med Sci Sports Exerc 1993; 25: 741-7.

11. Raglin JS, Morgan WP. Development of a scale for use in monitoring training-induced distress in athletes. Int J Sports Med. 1994; 15: 84-8.

12. Fry RW, Morton AR, Keast D: Overtraining in athletes. An update. Sports Med 1991; 12: 32-65.

13. Gleeson M. Biochemical and immunological markers of overtraining. Journal of Sports Science and Medicine 2002: 1: 31-41.

14. Houmard JA. Impact of reduced training on performance in endurance athletes. Sports Med 1991; 12: 380-93

15. Mackinnon LT. Special feature for the Olympics: effects of exercise on the immune system: overtraining effects on immunity and performance in athletes. Immunol Cell Biol 2000; 78: 502-9.

16. Armstrong LE, VanHeest JL. The unknown mechanism of the overtraining syndrome. Sports Med 2002: 32: 185-209

17. Peluso MAM. Alterações de humor associadas a atividade física intensa [tese]. São Paulo (SP): Universidade de São Paulo; 2003.

18. Terry PC. The efficacy of mood state profiling among elite performers: A review and synthesis. The Sport Psychologist 1995; 9: 309-24.
19. Morgan WP, Brown DR, Raglin JS, O'Connor PJ, Ellickson KA. Psychological monitoring of overtraining and staleness. Br J Sports Med 1987; 25: 107-14.

20. Terry PC, Lane AM, Lane HJ, Keohane L. Development and validation of a mood measure for adolescents. J Sports SC 1999; 17: 861-72.

21. Terry PC, Lane AM, Fogarty GJ. Construct validity of the POMS-A for use with adults. Psychology of Sport and Exercise 2003; 4: 125-39.

22. Terry, PC. Introduction to perspectives on mood in sport and exercise. Journal of Applied Sport Psychology 2000; 12: 1-5.

23. Weinberg, R S, Gould D. Fundamentos da Psicologia do Esporte e do Exercício. Tradução de Maria Cristina Morateiro. 2. ed. Porto Alegre: Artmed; 2001.

24. Rohlfs ICPM, Rotta TM, Andrade A, Terry PC, Krebs RJ, Carvalho T. The Brunel of mood scale (BRUMS): instrument for detection of modified mood states in adolescents and adults athletes and non athletes. Fiep Bulletin 2005; 75: 281-4.

25. Pasquali, L. (Org) Instrumentos psicológicos: manual prático de elaboração. Brasília: Lab PAM / IBAPP; 1999.

26. Menezes PR. Validade e confiabilidade das escalas de avaliação em psiquiatria. Rev Psiq Clin 1998: 25: $214-6$.

27. Armstrong BK, White E.; Saracci R. - Principles of Exposure Measurement in Epidemiology. Oxford, New York, Tokio: Oxford University Press; 1994.

28. Andrade A. Ocorrência e controle subjetivo do stress na percepção de bancários ativos e sedentários: a importância do sujeito na relação "atividade física e saúde" [tese]. Florianópolis(SC): Universidade Federal de Santa Catarina; 2001

29. Beck AT, Clark, DA. Anxiety and depression: an information processing perspective. Anxiety Research, 1988; 1: 23-56.

30. Spielberger CD. Manual for the State-Trait Anger-expression Inventory. Odessa, FL: Psychological Assessment Resources; 1991

31. Lane AM, Terry PC. The nature of mood: development of a conceptual model with a focus on depression. Journal of Applied Sport Psychology 2000; 12: 16-33.

32. Guillermin F, Bombardier C, Beaton D. Cross-cultural adaptation of health-related quality of life measures: literature review and proposed guidelines. J Clin Epidemiol 1993; 46: 1417-32.

33. Bardin, L. Análise de conteúdo. Lisboa: Edições 70; 1977.

34. Andrade LHSG, Gorenstein C. Aspectos gerais das escalas de avaliação de ansiedade. Rev Psiq Clin 1998; 25: 285-96 